ARTICLE

DOI: $10.1038 / s 41467-018-07143-8$

\title{
Unveiling a magnetized jet from a low-mass protostar
}

Chin-Fei Lee (10 1,2, Hsiang-Chih Hwang ${ }^{2,3}$, Tao-Chung Ching ${ }^{4}$, Naomi Hirano ${ }^{1}$, Shih-Ping Lai ${ }^{4}$, Ramprasad Rao ${ }^{1} \&$ Paul.T.P Ho ${ }^{1,5}$

Protostellar jets are one of the most intriguing signposts in star formation. Recent detection of a jet rotation indicates that they can carry away angular momenta from the innermost edges of the disks, allowing the disks to feed the central protostars. In current jet-launching models, magnetic fields are required to launch and collimate the jets, however, observationally, it is still uncertain if magnetic fields are really present in the jets. Here we report a clear detection of $\mathrm{SiO}$ line polarization in the $\mathrm{HH} 211$ protostellar jet. Since this line polarization has been attributed to the Goldreich-Kylafis effect in the presence of magnetic field, our observations show convincingly the presence of magnetic field in a jet from a low-mass protostar. The implied magnetic field could be mainly toroidal, as suggested in current jetlaunching models, in order to collimate the jet at large distances.

\footnotetext{
${ }^{1}$ Academia Sinica Institute of Astronomy and Astrophysics, P.O. Box 23-141, Taipei 106, Taiwan. ${ }^{2}$ Graduate Institute of Astronomy and Astrophysics, National Taiwan University, No. 1, Sec. 4, Roosevelt Road, Taipei 10617, Taiwan. ${ }^{3}$ Department of Physics and Astronomy, Johns Hopkins University, Baltimore, MD 21218, USA. ${ }^{4}$ Institute of Astronomy and Department of Physics, National Tsing Hua University, Hsinchu 30013, Taiwan. ${ }^{5}$ East Asian Observatory, 660N. A'ohoku Place, University Park, Hilo, HI 96720, USA. Correspondence and requests for materials should be addressed to C.-F.L. (email: cflee@asiaa.sinica.edu.tw)
} 
$\mathrm{P}$ rotostellar jets are believed to be the key to removing angular momenta from the innermost edges of the accretion disks, allowing the disk material to fall onto the protostars during the star formation. Recent detection of a rotation in a protostellar jet at high angular and velocity resolutions strongly supports this role of the jets ${ }^{1}$. In current jet-launching models, the jets are launched from the accretion disks by magnetic fields through magneto-centrifugal force ${ }^{2,3}$. In these models, poloidal fields are required to launch the jets and toroidal fields are required to collimate the jets. Therefore, the jets are expected to be magnetized. However, there are still no observations showing convincingly the presence and morphology of magnetic fields in the jets.

In a few protostellar jets from massive protostars, for example, $\mathrm{HH} 80-81^{4}$ and $\mathrm{W} 3(\mathrm{OH})^{5}$, their centimeter radio emission has been interpreted as synchrotron radiation because of its negative spectral index, as seen in the jets from active galactic nuclei (AGNs). Since synchrotron radiation is emitted by relativistic electrons in the presence of magnetic fields, this interpretation of radio emission suggests that the jets are magnetized. Detection of polarized radio emission can further support this interpretation, because synchrotron radiation is expected to be highly polarized. In addition, the observed polarization pattern can be used to derive the magnetic field morphology. So far, polarized radio emission was only detected in $\mathrm{HH} 80-81^{6}$. It was detected towards the jet-like structures at a large $(\gtrsim 30,000$ a.u.) distance from the central protostar at a spatial resolution of $\sim 20,000$ a.u., with the polarization orientations roughly perpendicular to the jet axis. The implied magnetic field there could be helical, similar to what is expected in the AGN jets. A recent study with observations at $\sim 8$ times higher resolution suggested that the synchrotron emission there arises from an extended component and from the termination points of the jet, where strong shocks against the ambient medium can produce efficient particle acceleration ${ }^{7}$.

The jets from low-mass protostars are less energetic than those from massive protostars, and thus only very few of them, for example, $\mathrm{L} 778 \mathrm{VLA}^{8}$ and DG Tau ${ }^{9}$, show non-thermal emission that could be synchrotron radiation. Fortunately, when they are young, they have high content of molecular gas because of high mass-loss rate ${ }^{10-13}$. Therefore, a plausible way to map the magnetic fields in these jets is to map the linear polarization raised by the molecules (e.g., $\mathrm{SiO}$ and $\mathrm{CO}$ ) themselves-the so-called Goldreich-Kylafis (GK) effect ${ }^{14,15}$, as was previously done for molecular outflows ${ }^{16}$. In the presence of a magnetic field, a molecular rotational level splits into magnetic sublevels, producing a line polarization with its orientation either parallel or perpendicular to the magnetic field. In general, maximum polarization occurs when the line of sight is perpendicular to the velocity flow and the magnetic field direction ${ }^{17}$. Therefore, jets lying close to the plane of sky have the highest chance to produce observable polarization from the GK effect.

HH 211 is a nearby jet in the Perseus molecular cloud at a distance recently updated to be $\sim 321 \pm 10 \mathrm{pc}^{18}$. It lies within $\sim 9^{\circ}$ of the plane of the $\mathrm{sky}^{19,20}$, providing the best chance to map for the magnetic field. It is powered by a very young Class 0 protostar, which has a mass of $\lesssim 0.05 M_{\odot} 21,22$ and is surrounded by a small rotating disk ${ }^{22}$, and thus has a high content of molecular gas. It is best seen in $\mathrm{SiO}$, appearing as a fast-moving highly collimated structure propagating down the jet axis inside the cavity of a slow-moving less-collimated outflow seen in $\mathrm{H}_{2}{ }^{23}$ (see Fig. 1) and $\mathrm{CO}^{19,24}$. It consists of the material coming out from the disk ${ }^{19,23}$ and thus is intrinsically different from the CO outflow that consists mostly of ambient material. The $\mathrm{SiO}$ emission of the jet is brightest at $J=8-7$ transition. At this transition, the optical depth is close to $1^{19}$ and the collision rate is lower than the radiative transition rate for a typical jet density of $10^{6}-10^{7} \mathrm{~cm}^{-3}$, both optimal for polarization from the GK effect ${ }^{14,15}$. Here we report our detection of $\mathrm{SiO}$ line polarization in the inner part of the jet with the Atacama Large Millimeter/submillimeter Array (ALMA). This is the first convincing polarization detection towards the jet of a low-mass protostar, and at much closer distances from the protostar than in previous studies.

\section{Results}

SiO line polarization in the $\mathrm{HH} 211$ jet. Figure $2 \mathrm{a}$ shows the mean intensity map of the jet in the inner part in $\mathrm{SiO} J=8-7$ at $0^{\prime \prime} .21 \times 0^{\prime \prime} .14$ (67 a.u. $\times 45$ a.u.) resolution. It shows the intensity averaged over a velocity range of $\sim 30 \mathrm{~km} \mathrm{~s}^{-1}$, in which the jet emission is detected, for both redshifted and blueshifted jet components. In our observations, the field of view (see Methods) is a circular region within $\sim 8^{\prime \prime}$ (i.e., 2600 a.u.) of the central source, covering the jet emission out to knots BK3 and RK3. The jet is knotty and highly collimated, as seen before. The linear structures within $\sim 3^{\prime \prime}$ of the central source are knots BK1 and $\mathrm{RK} 1$, and they are now each resolved into a few subknots, for example, $\mathrm{A}$ and $\mathrm{B}$ in knot $\mathrm{BK} 1$, as shown in Fig. 2b. The jet is marginally resolved in the transverse direction. The cylindrical radius of the jet, if assumed to be a half of the Gaussian deconvolved width of the subknots, is estimated to be $\lesssim 25$ a.u. (i.e., $0 " .08$, similar to that found before at a similar resolution ${ }^{19}$ ).

In the blueshifted jet component (blue contours), polarized $\mathrm{SiO}$ emission is clearly detected with $4 \sigma$ to $7 \sigma$ at the two brightest subknots, A and B, within $\sim 1^{\prime \prime} .5$ ( $\sim 480$ a.u.) of the central source and $3 \sigma$ to $4 \sigma$ at some other spots further out (see Fig. 2b). The polarization degree is about 1 to $2 \%$, with a mean of $\sim 1.5 \%$, as shown by the length of the line segments in Fig. $2 \mathrm{c}$. In addition, subknot A has a higher polarization degree than subknot B, probably because its optical depth is closer to $1^{15}$. Interestingly, all the polarization orientations are almost parallel to the jet axis. We can also map the polarization of the two subknots with enough signal-to-noise ratio in two wide-velocity channels with a width of $\sim 14 \mathrm{~km} \mathrm{~s}^{-1}$, as shown in Fig. 3. As can be seen, the polarization orientations and polarization degrees of the subknots in these two velocity channels are roughly the same and similar to those seen in Fig. 2c, which is obtained by averaging over the whole velocity range. This suggests that the polarization orientations and degrees are roughly the same at different velocities. Further observations with enough sensitivity in narrower velocity channels are needed to confirm this.

In the redshifted jet component (red contours in Fig. 2), the $\mathrm{SiO}$ emission is weaker than that in the blueshifted jet component. Assuming the same polarization degree of $\sim 1.3 \%$ as in subknot $\mathrm{B}$ in the blueshifted jet component, the polarized intensity at the brightest subknots (peaks) is expected to be $\sim 2$ $\mathrm{mJy}$ per beam, which is only $\sim 3 \sigma$. Also, since the subknots have a size much smaller than the maximum recoverable scale $\left(\sim 1^{\prime \prime} .1\right.$, see Methods) in our observations, the polarized emission, if it exists towards the subknots, will not be resolved out. Thus, deeper observations are needed to detect $\mathrm{SiO}$ line polarization in the redshifted jet component.

Our observations provide the first reliable detections of $\mathrm{SiO}$ line polarization in the protostellar jet, with high enough signalto-noise ratios. Previously at four times lower resolution with the Submillimeter Array, we had two tentative detections of SiO line polarization in the outer part of the jet, one at knot RK2 and the other in between knot BK2 and BK3 $3^{25}$. The one at knot RK2 has a polarization orientation parallel to the jet axis, as seen here in the inner part of the blueshifted jet component, but with a much higher polarization degree of $\sim 10 \%$. The one in between knots $\mathrm{BK} 2$ and $\mathrm{BK} 3$, where the $\mathrm{SiO}$ emission is faint, has a polarization degree of $\gtrsim 20 \%$ and an orientation inclined by $\sim 50^{\circ}$ to the jet 


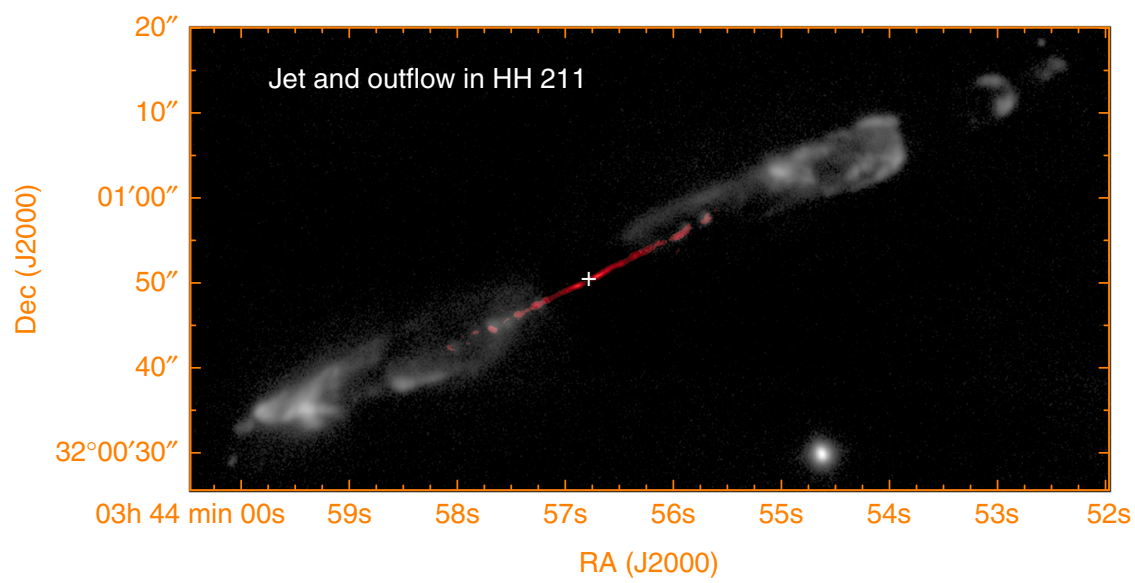

Fig. 1 Jet and outflow in $\mathrm{HH}$ 211. Gray image shows the outflow and the outer part of the jet in $\mathrm{H}_{2}$ adopted from Hirano et al.23. Red image shows the inner part of the jet in SiO adopted from Lee et al ${ }^{19}$. The cross marks the position of the central driving source at $\alpha_{(2000)}=03^{\mathrm{h}} 43^{\mathrm{m}} 56 .{ }^{\mathrm{s}} 8053$,

$\delta_{(2000)}=32^{\circ} 00^{\prime} 50 .^{\prime \prime} 192^{22}$

RA (J2000)
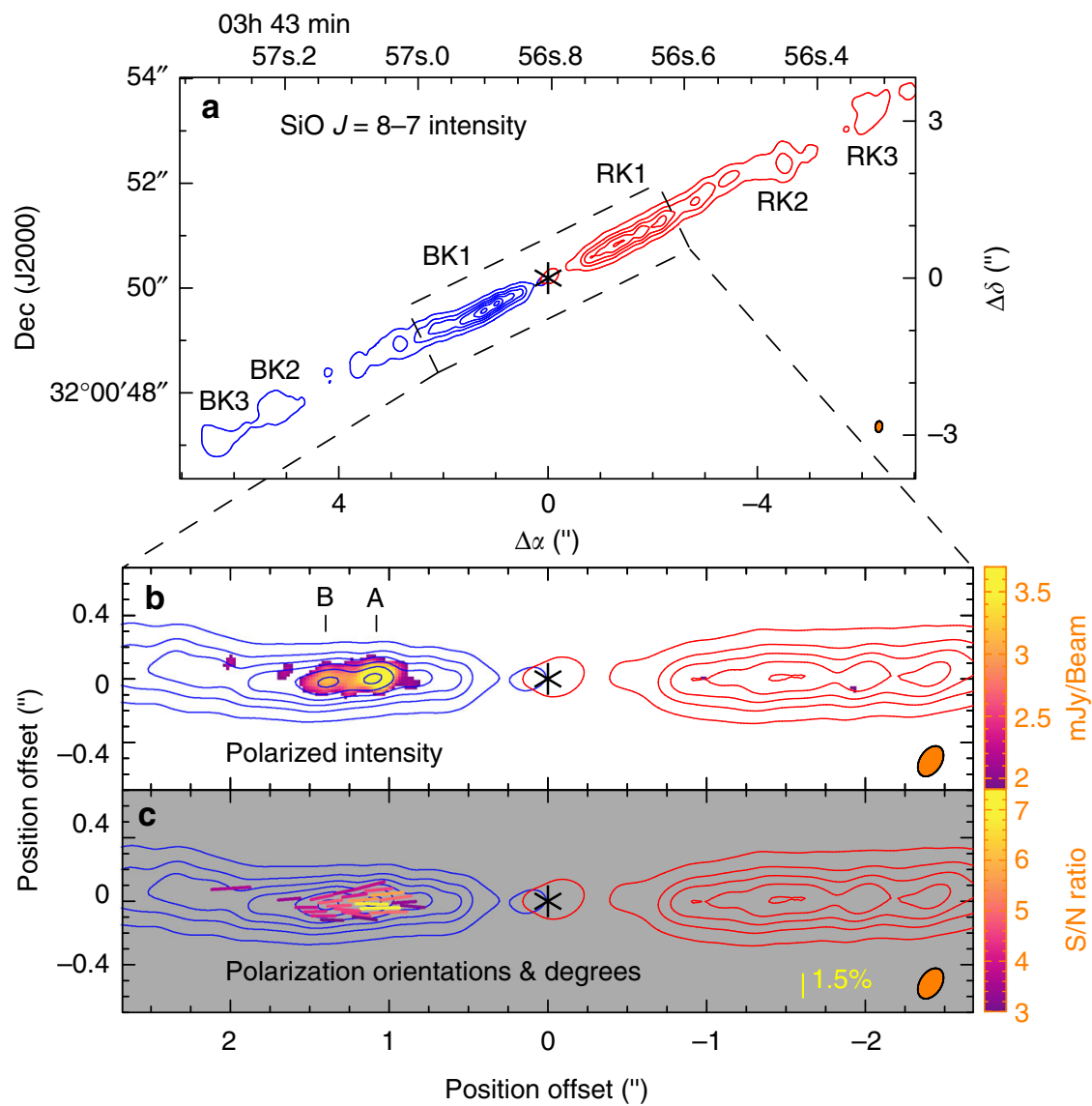

Fig. 2 a Polarization results towards the inner part of the $\mathrm{HH} 211$ jet in $\mathrm{SiO} J=8-7$. The resolution is $0^{\prime \prime} .21 \times 0$ ".14, as shown with the elliptical beams in the bottom right corners. Asterisks mark the source position. In a, knots BK1, BK2, BK3, RK1, RK2, and RK3 are the SiO knots identified before ${ }^{11}$, 19 . Subknots $A$ and $B$, where polarized emission is detected, are the two subknots in knot BK1 resolved in this work. In $\mathbf{b}$, $\mathbf{c}$, the image of the jet is rotated by $26.6^{\circ}$ clockwise to be aligned with the $x$-axis. The blueshifted component (blue contours) is obtained by averaging the emission from -22 to $8 \mathrm{~km} \mathrm{~s}^{-1}$, and the redshifted component (red contours) from 11 to $42 \mathrm{~km} \mathrm{~s}^{-1}$. The contours start from $30 \sigma$ with a step of $60 \sigma$, where $\sigma \sim 0.64 \mathrm{mJy}$ per beam, which is the noise level of polarized emission. $\mathbf{b}$ The color image shows the polarized intensity $>3 \sigma$ detection. $\mathbf{c}$ Line segments show the polarization orientations ( $E$ vectors), with their length indicating the polarization degree. They are color coded according to their $\mathrm{S} / \mathrm{N}$ ratio in polarized intensity 


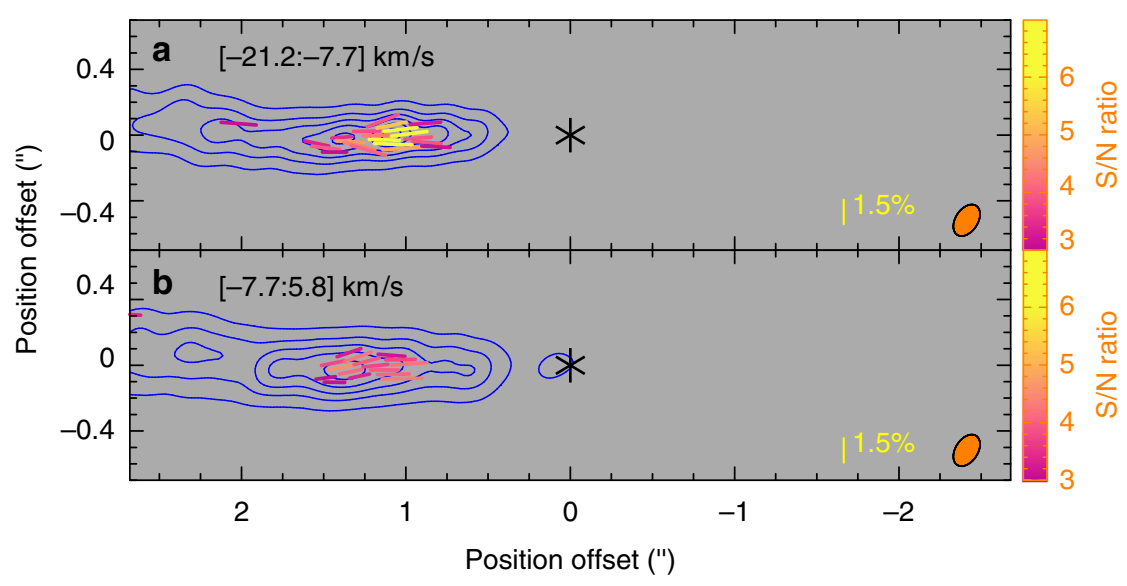

Fig. $3 \mathrm{SiO}$ line polarization results in the blueshifted component of the $\mathrm{HH} 211$ jet in two wide-velocity channels. The velocity ranges are indicated in the upper left corners. The asterisk marks the position of the central driving source. Contours show the intensity of the $\mathrm{SiO}$ emission. They start from $30 \sigma$ with a step of $60 \sigma$, where $\sigma \sim 0.85 \mathrm{mJy}$ per beam, which is the noise level of polarized emission. Line segments show the polarization orientations ( $E$ vectors), with their length indicating the polarization degree. They are color coded according to their $\mathrm{S} / \mathrm{N}$ ratio in polarized intensity

axis. Since those detections were only at $\sim 3 \sigma$ level, further observations are needed to confirm them. Unfortunately, we cannot confirm those detections because we do not detect any polarized emission towards those knots. Notice that those knots are located more than 3 " away from the central source and thus outside the region where the polarization can be mapped properly in our observations (see Methods).

Magnetic field morphology in the HH 211 jet. SiO line polarizations have been detected in the circumstellar envelopes of evolved stars, with a maximum polarization degree of $\sim 4 \%^{26}$, higher than that seen here in the jet, probably because of different physical environments and velocity structures. They were attributed to the GK effect and thus used to map the magnetic field morphology in the circumstellar envelopes of evolved stars. Similarly, $\mathrm{SiO}$ line polarization detected here can be used to infer the magnetic field morphology in the jet. Here the polarization orientations are all almost aligned with the jet axis and thus the velocity flow axis. In this case, according to the GK effect, the magnetic fields could be either parallel or perpendicular to the polarization orientations ${ }^{27}$, and thus could be either poloidal or toroidal.

One way to resolve this ambiguity in field morphology is to map one additional $\mathrm{SiO}$ line, because the polarization direction and relative polarization degree in two $\mathrm{SiO}$ lines could be used to judge the magnetic field direction, as done before with two CO lines for the $\mathrm{CO}$ outflows in other sources ${ }^{16,28}$. Another way to resolve the ambiguity is to compare the polarized $\mathrm{SiO}$ emission with polarized dust continuum emission in the same location, as done before to resolve the field ambiguity in the CO outflows in other sources ${ }^{16,29,30}$. Unfortunately, in our ALMA observations of $\mathrm{HH} 211$, the dust emission at $350 \mathrm{GHz}$, which traces the envelope and disk, is only detected within $\sim 0^{\prime \prime} .4$ of the central source, without any overlap with the polarized $\mathrm{SiO}$ emission in the jet, as shown in Fig. 4. Previous observation of $\mathrm{HH} 211$ at $\sim 4^{\prime \prime}$ resolution ${ }^{31}$ showed extensions of dust continuum emission at $230 \mathrm{GHz}$ along the outflow axis. Those extensions appear because of the accidental inclusion of CO emission in their map (C. Hull, private communication) and are thus probably from the $\mathrm{CO}$ outflow around the $\mathrm{SiO}$ jet. Further observations at higher sensitivity in continuum are needed to check if the dust emission can trace the jet and thus can be used to resolve the field ambiguity in the jet.

\section{Discussion}

Protostellar jets are generally thought to be launched from accretion disks ${ }^{32}$. In particular, two competing models, the $\mathrm{X}$ wind model $^{2}$ and disk-wind model $^{3}$, have been constructed to launch the jets from the disks through the magneto-centrifugal force. The jets are launched from the disks at a radius of $\sim 0.05 \mathrm{a}$. $\mathrm{u}$. in the $\mathrm{X}$-wind model $^{2}$, while from $\sim 0.1$ a.u. to a few a.u. in the disk-wind model $^{3}$. In these two models, the magnetic fields in the jets at a distance much farther than the launching radius are expected to be mainly toroidal in order to collimate the jets ${ }^{2,3}$. Therefore, the magnetic fields at subknots $A$ and $B$, which are at a distance of $\sim 350$ and 460 a.u. and thus much farther than the launching radius, should be mainly toroidal. If this is the case, the implied magnetic field orientations here would be perpendicular to the observed polarization orientations, as shown in Fig. 5. Since the observed orientations of the polarization give the projections of the magnetic fields on the plane of the sky, the magnetic fields here could also be helical, as suggested in the $\mathrm{HH} \mathrm{80-81} \mathrm{jet} \mathrm{from}$ the massive protostar and in the AGN jets, but tightly wound. Further observations are needed to confirm this.

The magnetic field strength can be estimated in the two subknots, $\mathrm{A}$ and $\mathrm{B}$, in the blueshifted jet component, where sufficient number of polarization vectors are detected. According to Chandrasekhar et al..$^{33}$ and Ostriker et al. ${ }^{34}$, the field strength in the plane of the sky can be estimated with the following equation:

$$
B \sim 0.5 \sqrt{4 \pi \rho} \frac{\Delta v_{\text {los }}}{\Delta \phi},
$$

where $\rho$ is the mass density, $\Delta v_{\text {los }}$ is the velocity dispersion along the line of sight, and $\Delta \phi$ is the dispersion of the polarization angle. According to Lee et al. ${ }^{35}$, the volume density in the jet $n_{\mathrm{H}_{2}} \gtrsim 2.0 \times 10^{6} \mathrm{~cm}^{-3}$ (updated for the new jet radius because of new distance), and thus $\rho=1.4 n_{\mathrm{H}_{2}} m_{\mathrm{H}_{2}} \gtrsim 9.3 \times 10^{-18} \mathrm{~g} \mathrm{~cm}^{-3}$. Also from Figure $8 \mathrm{a}$ in Lee et al. ${ }^{19}$, we have $\Delta v_{\text {los }} \sim 15 \mathrm{~km} \mathrm{~s}^{-1}$, which is the full width at half-maximum of the $\mathrm{SiO}$ velocity distribution towards knot BK1. This velocity width can be produced by a turbulent motion due to localized shocks in the knot. From our observations, we have $\Delta \phi \sim 30^{\circ} \pm 20^{\circ}$, and thus $B \gtrsim 15_{-6}^{+30} \mathrm{mG}$.

Is this magnetic field strength reasonable? Answering this question requires us to know which jet-launching model is more appropriate for $\mathrm{HH} 211$. Previously, a hint of jet rotation was reported in $\mathrm{HH} 211^{19}$. However, our observations here with 


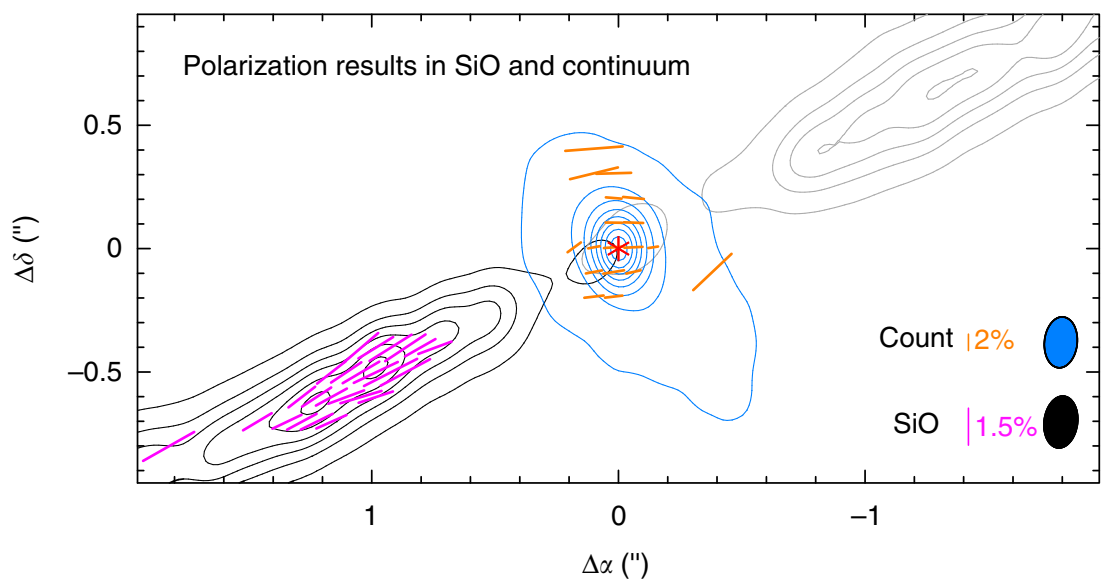

Fig. 4 A comparison of polarized SiO emission with polarized dust continuum emission in $\mathrm{HH}$ 211. The asterisk marks the position of the central driving source. Black and gray contours show the blueshifted and redshifted jet components, respectively, with their contour levels the same as in Fig. 2. Magenta line segments show the polarization orientations of the polarized $\mathrm{SiO}$ emission. Blue contours and orange line segments show the intensity and the orientations of the polarized emission in continuum at $350 \mathrm{GHz}$, obtained alongside in our ALMA observations (Lee et al., in preparation)

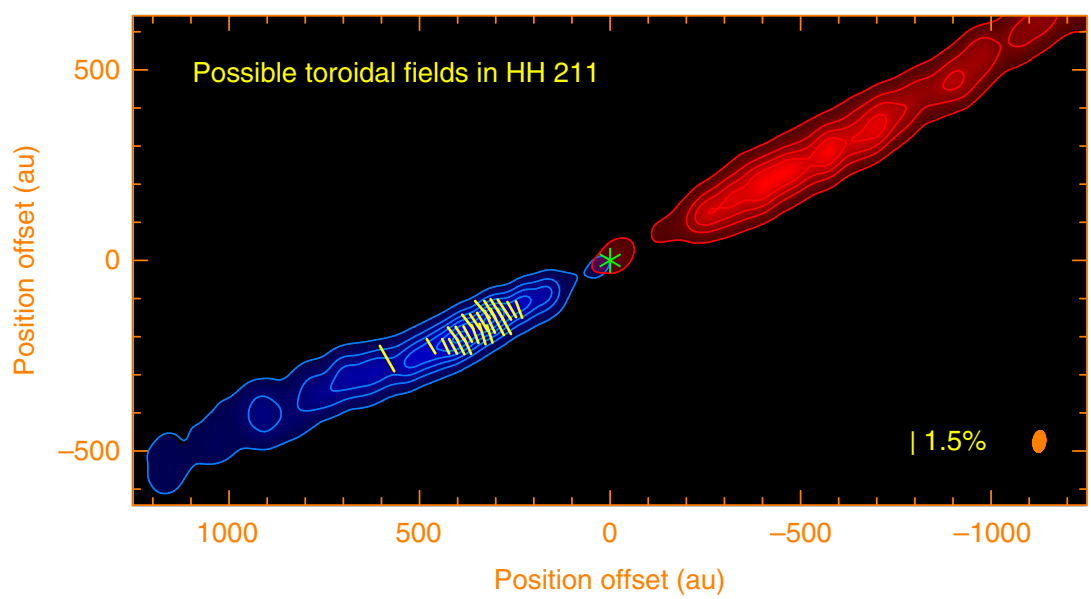

Fig. 5 Possible toroidal fields in the $\mathrm{HH} 211$ jet. Yellow line segments indicate the possible toroidal fields obtained by rotating the polarization orientations by $90^{\circ}$. The asterisk marks the position of the central driving source. Blue image with the blue contours shows the blueshifted jet component. Red image with the red contours shows the redshifted jet component. The contour levels are the same as in Fig. 2

\section{Table 1 Correlator setup}

\begin{tabular}{|llllll} 
Spectral window & Line or continuum & Number of channels & Central frequency $(\mathbf{G H z})$ & Bandwidth (MHz) & Channel width (kHz) \\
\hline 0 & $\mathrm{SO} \mathrm{NJ}=8_{9}-7_{8}$ & 480 & 346.533 & 117.187 & 244.141 \\
1 & $\mathrm{CO} \mathrm{J=3-2}$ & 480 & 345.800 & 117.187 & 244.141 \\
2 & $\mathrm{SiO} J=8-7$ & 960 & 347.335 & 468.750 & 488.281 \\
3 & $\mathrm{HCO} J=4-3$ & 960 & 356.739 & 234.375 & 244.141 \\
4 & Continuum & 960 & 358.005 & 1875.000 & 1953.125 \\
\hline
\end{tabular}

similar resolution but much higher sensitivity found no clear rotation. Therefore, with the velocity $\left(\sim 0.42 \mathrm{~km} \mathrm{~s}^{-1}\right.$ per channel) and spatial ( $\sim 64$ a.u.) resolutions in our observations, we can assume an upper limit of $\sim 27$ a.u. $\mathrm{km} \mathrm{s}^{-1}$ for the jet-specific angular momentum. Then, using a jet velocity 20,35 of $\sim 150 \mathrm{~km} \mathrm{~s}^{-1}$ and a protostellar mass of $\sim 0.05 M_{\odot}^{22}$, the jet-launching radius is estimated to be $\lesssim 0.06$ a.u. ${ }^{36}$ and thus more consistent with the Xwind model. In a typical $\mathrm{X}$-wind model for a low-mass protostellar jet-like HH 211, the magnetic field strength is expected to be $\gtrsim 6 \mathrm{mG}$ for a jet cylindrical radius of $\lesssim 25$ a.u. ${ }^{37}$. Hence, the estimated field strength from our observations is about 2.5 times as high as the expected, probably reasonable considering a shock compression in the subknots and all the uncertainties in the measurements. The resulting magnetic pressure will be $B^{2} / 8 \pi \gtrsim$ $8.9 \times 10^{-6}$ dyne $\mathrm{cm}^{-2}$. This is much higher than the thermal pressure in the subknots, which is $1.2 n_{\mathrm{H}_{2}} k T \gtrsim 1.7 \times 10^{-7}$ dyne 
$\mathrm{cm}^{-2}$ with a density $n_{\mathrm{H}_{2}} \sim 2.0 \times 10^{6} \mathrm{~cm}^{-3}$ and the highest possible $T \sim 500 \mathrm{~K}^{23}$. As a result, the magnetic field, if toroidal, is strong enough to confine and thus collimate the jet material.

In conclusion, we have detected convincingly polarized $\mathrm{SiO}$ line emission in the protostellar jet, although deeper observations are needed to detect the polarization in the redshifted component of the jet and check for consistent morphology of the polarization. Our detection has opened up an opportunity to use the GK effect to map the magnetic field morphology in the jet in the early phase of star formation. Based on the current jet-launching models, the implied magnetic field should be mainly toroidal at large distance. In the future, we will follow-up with polarization observations in $\mathrm{SiO} J=5-4$ in order to confirm the field morphology.

\section{Methods}

Observations. Polarization observations of the $\mathrm{HH} 211$ protostellar jet were carried out with ALMA in Band 7 at $\sim 350 \mathrm{GHz}$ in Cycle 4, with 43 antennas in C40-7 configuration. The project number was 2016.1.00017.S. The phase center was $03^{\mathrm{h}} 43^{\mathrm{m}} 56 .^{\mathrm{s}} 8040 \delta_{(2000)}=32^{\circ} 00^{\prime} 50 .^{\prime \prime} 270$, but the maps here are produced and presented with a center at the central source position at $\alpha_{(2000)}=03^{\mathrm{h}} 43^{\mathrm{m}} 56^{\mathrm{s}} 8053$, $\delta_{(2000)}=32^{\circ} 00^{\prime} 50$. $^{\prime \prime} 192^{22}$. Two executions were carried out on 10 October 2016, with a total time of $\sim 77 \mathrm{~min}$ on the $\mathrm{HH} 211$ jet. The projected baselines are $\sim 20-1500 \mathrm{~m}$. One pointing, with a primary beam (field of view) of $\sim 17^{\prime \prime}$ covering a circular region within $\sim 8^{\prime \prime}$ of the central source, was used to map the inner part of the jet. The maximum recoverable scale is $\sim 1^{\prime \prime} .1$. Since the jet consists of a chain of knots and subknots, the detection of polarized emission towards the subknots, which have a size of $\sim 0^{\prime \prime} .2$, will not be affected. However, since the jet seems to have a smooth structure greater than the maximum recoverable scale, the polarized emission there, if exists, could be partially resolved out.

The correlator was set up to have five spectral windows, with one for SO $N_{J}=$ $8_{9}-7_{8}$ at $346.528481 \mathrm{GHz}$, one for CO $J=3-2$ at $345.795991 \mathrm{GHz}$, one for $\mathrm{SiO} J$ $=8-7$ at $347.330631 \mathrm{GHz}$, one for $\mathrm{HCO}^{+} J=4-3$ at $356.734288 \mathrm{GHz}$, and one for the continuum at $358 \mathrm{GHz}$ (see Table 1). In this paper, we only present the observational results in $\mathrm{SiO}$, which traces uniquely the jet emanating from the central source. The velocity resolution is $0.42 \mathrm{~km} \mathrm{~s}^{-1}$ per channel in SiO. The data were calibrated with the CASA package (versions 4.7), with Quasar J0238 +1636 as a flux calibrator, Quasar J0237 $+2848(\sim 0.833 \mathrm{Jy})$ as a passband calibrator, Quasar $\mathrm{J} 0336+3218(\sim 0.517 \mathrm{Jy})$ as a gain calibrator, and Quasar J0334 - $4008(\sim 0.415 \mathrm{Jy})$ as a polarization calibrator. A phase-only self-calibration of the data was performed to improve the map fidelity using the continuum intensity (Stokes $I$ ) map towards the central source. We used a robust factor of 2 for the visibility weighting to generate the $\mathrm{SiO}$ maps (including Stokes $I, Q$, and $U$ parameters) at a resolution of $\sim 0^{\prime \prime} .2$. No primary beam correction is applied to the maps here because we only focus on the inner part of the jet where the polarized emission can be mapped properly (see below). In addition, no polarization is detected beyond $2^{\prime \prime}$ of the central source. In order to detect the polarization at sufficient sensitivity, we made the mean intensity maps for the redshifted jet component and blueshifted jet component (see Fig. 2), by averaging the emission intensity over a velocity range of $\sim 30 \mathrm{~km} \mathrm{~s}^{-1}$, in which the jet emission is detected. The noise level is $\sim 2.73 \mathrm{mJy}$ per beam in the Stokes $I$ map and $\sim 0.64 \mathrm{mJy}$ per beam in the Stokes $Q$ and $U$ maps for the SiO line. In order to check for any polarization dependence on velocity in the blueshifted jet component where the polarization is well detected, we also present polarization maps in two wide-velocity channels with a velocity width of $\sim 14 \mathrm{~km} \mathrm{~s}^{-1}$. The noise level is $\sim 0.85 \mathrm{mJy}$ per beam in the Stokes $Q$ and $U$ maps of those wide channels.

Polarization orientations are defined by the $E$ vectors. Linear polarization degree (fraction) is defined as $P=\sqrt{Q^{2}+U^{2}} / I$. According to ALMA Technical Handbook in Cycle 4, polarization imaging with accuracy better than $0.3 \%$ can be achieved within the inner $1 / 3$ of the primary beam and this determines the largest acceptable angular size of sources which can be observed in full polarization. Therefore, in our observations with the phase center at the source position, we can only map the polarization of the jet reliably within $\sim 3^{\prime \prime}$ of the central source.

\section{Data availability}

This article makes use of the following ALMA data: ADS/JAO.ALMA\#2016.1.00017.S. The data that support the plots within this paper and other findings of this study are available from the corresponding author upon reasonable request.

Received: 13 July 2018 Accepted: 17 October 2018

Published online: 06 November 2018

\section{References}

1. Lee, C.-F. et al. A rotating protostellar jet launched from the innermost disk of HH 212. Nat. Astron. 1, 0152 (2017).

2. Shu, F. H., Najita, J. R., Shang, H. \& Li, Z.-Y. X-winds theory and observations. Prot. Planets IV, 789-814 (2000).

3. Pudritz, R. E., Ouyed, R., Fendt, C., \& Brandenburg, A. in Protostars and Planets V (eds Reipurth, B., Jewitt, D. \& Keil, K.) 277-294 (University of Arizona Press, Tucson, 2007)

4. Marti, J., Rodriguez, L. F. \& Reipurth, B. HH 80-81: a highly collimated Herbig-Haro complex powered by a massive young star. ApJ 416, 208 (1993).

5. Wilner, D. J., Reid, M. J. \& Menten, K. M. The synchrotron jet from the $\mathrm{H}_{2} \mathrm{O}$ maser source in W3(OH). ApJ 513, 775 (1999).

6. Carrasco-González, C. et al. A magnetized jet from a massive protostar. Science 330, 1209 (2010).

7. Rodrguez-Kamenetzky, A. et al. The highly collimated radio jet of $\mathrm{HH} 80-81$ : structure and nonthermal emission. ApJ 851, 16 (2017).

8. Girart, J. M., Curiel, S., Rodrguez, L. F. \& Cantó, J. Radio continuum observations towards optical and molecular outflows. Rev. Mex. Astron. Astro. 38, 169 (2002).

9. Ainsworth, R. E. et al. Tentative evidence for relativistic electrons generated by the jet of the young sun-like star DG Tau. ApJL 792, L18 (2014).

10. Glassgold, A. E., Mamon, G. A. \& Huggins, P. J. The formation of molecules in protostellar winds. ApJ 373, 254-265 (1991).

11. Lee, C.-F. et al. Submillimeter arcsecond-resolution mapping of the highly collimated protostellar jet HH 211. ApJ 670, 1188 (2007).

12. Hirano, N. et al. Extreme active molecular jets in L1448C. ApJ 717, 58 (2010)

13. Lee, C.-F. et al. Jet motion, internal working surfaces, and nested shells in the protostellar system HH 212. ApJ 805, 186 (2015).

14. Goldreich, P. \& Kylafis, N. D. On mapping the magnetic field direction in molecular clouds by polarization measurements. ApJL 243, L75 (1981).

15. Goldreich, P. \& Kylafis, N. D. Linear polarization of radio frequency lines in molecular clouds and circumstellar envelopes. ApJ 253, 606 (1982).

16. Ching, T.-C. et al. Helical magnetic fields in the NGC 1333 IRAS 4A protostellar outflows. ApJ 819, 159 (2016).

17. Deguchi, S. \& Watson, W. D. Linear polarization of molecular lines at radio frequencies. ApJ 285, 126 (1984).

18. Ortiz-León, G. N. et al. The Gould's Belt Distances Survey (GOBELINS). V. Distances and kinematics of the Perseus molecular cloud. ApJ arXiv:1808 03499 (2018)

19. Lee, C.-F. et al. Rotation and outflow motions in the very low-mass class 0 protostellar system HH 211 at subarcsecond resolution. ApJ 699, 1584 (2009).

20. Jhan, K.-S. \& Lee, C.-F. A Multi-Epoch SMA Study of the HH 211 protostellar jet: jet motion and knot formation. ApJ 816, 32 (2016).

21. Froebrich, D., Smith, M. D., Hodapp, K.-W. \& Eislöffel, J. Far-infrared photometry of deeply embedded outflow sources. MNRAS 346, 163 (2003).

22. Lee, C.-F. et al. ALMA observations of the very young class 0 protostellar system HH211-mms: a 30 au dusty disk with a disk wind traced by SO? ApJ 863, 94 (2018).

23. Hirano, N. et al. $\mathrm{SiO} J=5-4$ in the $\mathrm{HH} 211$ protostellar jet imaged with the submillimeter array. ApJL 636, L141 (2006).

24. Gueth, F. \& Guilloteau, S. The jet-driven molecular outflow of HH 211. A\&A 343, 571 (1999).

25. Lee, C.-F. et al. Magnetic field structure in the flattened envelope and jet in the young protostellar system HH 211. ApJL 797, L9 (2014).

26. Vlemmings, W. H. T. et al. Magnetically aligned dust and $\mathrm{SiO}$ maser polarisation in the envelope of the red supergiant VY Canis Majoris. A\&A 603, A92 (2017).

27. Kylafis, N. D. Polarization of interstellar radio-frequency lines and magnetic field direction. ApJ 267, 137 (1983)

28. Cortes, P. C., Crutcher, R. M. \& Watson, W. D. Line polarization of molecular lines at radio frequencies: the case of DR 21(OH). ApJ 628, 780 (2005).

29. Lai, S.-P., Velusamy, T. \& Langer, W. D. The high angular resolution measurement of ion and neutral spectra as a probe of the magnetic field structure in DR 21(OH). ApJL 596, L239 (2003).

30. Girart, J. M., Crutcher, R. M. \& Rao, R. Detection of polarized CO emission from the molecular outflow in NGC 1333 IRAS 4A. ApJL 525, L109 (1999).

31. Hull, C. L. H. et al. TADPOL: a $1.3 \mathrm{~mm}$ survey of dust polarization in starforming cores and regions. ApJS 213, 13 (2014).

32. Frank, A. et al. Jets and outflows from star to cloud: observations confront theory. Prot. Planets VI, 451 (2014).

33. Chandrasekhar, S. \& Fermi, E. Magnetic fields in spiral arms. ApJ 118, 113 (1953).

34. Ostriker, E. C., Stone, J. M. \& Gammie, C. F. Density, velocity, and magnetic field structure in turbulent molecular cloud models. ApJ 546, 980 (2001).

35. Lee, C.-F. et al. The reflection-symmetric wiggle of the young protostellar jet HH 211. ApJ 713, 731 (2010).

36. Anderson, J. M., Li, Z.-Y., Krasnopolsky, R. \& Blandford, R. D. Locating the launching region of T Tauri winds: the case of DG Tauri. ApJL 590, L107 (2003). 
37. Shu, F. H., Najita, J., Ostriker, E. C. \& Shang, H. Magnetocentrifugally driven flows from young stars and disks. V. Asymptotic collimation into jets. ApJL 455, L155 (1995).

\section{Acknowledgements}

This paper makes use of the following ALMA data: ADS/JAO.ALMA\#2016.1.00017.S. ALMA is a partnership of ESO (representing its member states), NSF (USA), and NINS (Japan), together with NRC (Canada), NSC and ASIAA (Taiwan), and KASI (Republic of Korea), in cooperation with the Republic of Chile. The Joint ALMA Observatory is operated by ESO, AUI/NRAO, and NAOJ. C.-F.L. acknowledges grants from the Ministry of Science and Technology of Taiwan (MoST 104-2119-M-001-015-MY3 and 1072119-M-001-040-MY3) and the Academia Sinica (Career Development Award).

\section{Author contributions}

C.-F.L. led the project, analysis, discussion, and drafted the manuscript. H.-C.H. produced a preliminary $\mathrm{SiO}$ polarization model from the GK effect to compare with our observations. C.-F.L., H.-C.H., T.-C.C., N.H., S.-P.L., R.R., and P.T.P.H. contribute to scientific discussion.

\section{Additional information}

Supplementary Information accompanies this paper at https://doi.org/10.1038/s41467018-07143-8.

Competing interests: The authors declare no competing interests.
Reprints and permission information is available online at http://npg.nature.com/ reprintsandpermissions/

Publisher's note: Springer Nature remains neutral with regard to jurisdictional claims in published maps and institutional affiliations.

(c) (i) Open Access This article is licensed under a Creative Commons Attribution 4.0 International License, which permits use, sharing, adaptation, distribution and reproduction in any medium or format, as long as you give appropriate credit to the original author(s) and the source, provide a link to the Creative Commons license, and indicate if changes were made. The images or other third party material in this article are included in the article's Creative Commons license, unless indicated otherwise in a credit line to the material. If material is not included in the article's Creative Commons license and your intended use is not permitted by statutory regulation or exceeds the permitted use, you will need to obtain permission directly from the copyright holder. To view a copy of this license, visit http://creativecommons.org/ licenses/by/4.0\%.

(c) The Author(s) 2018 